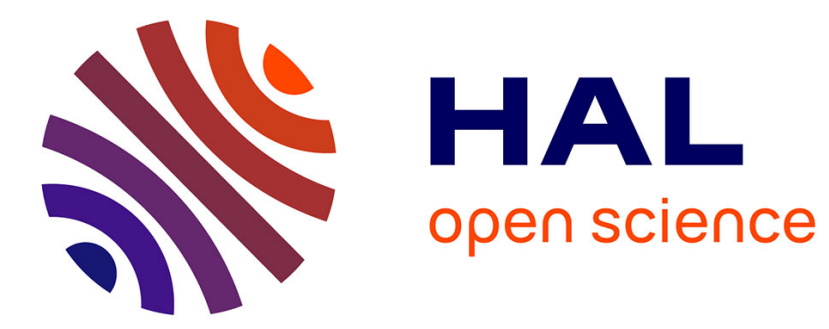

\title{
GEOMETRIC BASED 3D FACIAL GENDER CLASSIFICATION
}

\author{
Lahoucine Ballihi, Boulbaba Ben Amor, Mohamed Daoudi, Anuj Srivastava, \\ Driss Aboutajdine
}

\section{- To cite this version:}

Lahoucine Ballihi, Boulbaba Ben Amor, Mohamed Daoudi, Anuj Srivastava, Driss Aboutajdine. GEOMETRIC BASED 3D FACIAL GENDER CLASSIFICATION. INTERNATIONAL SYMPOSIUM ON COMMUNICATIONS, CONTROL, AND SIGNAL PROCESSING, May 2012, Rome, Italy. pp.15, 10.1109/ISCCSP.2012.6217828 . hal-00726221

\section{HAL Id: hal-00726221 \\ https://hal.science/hal-00726221}

Submitted on 29 Aug 2012

HAL is a multi-disciplinary open access archive for the deposit and dissemination of scientific research documents, whether they are published or not. The documents may come from teaching and research institutions in France or abroad, or from public or private research centers.
L'archive ouverte pluridisciplinaire HAL, est destinée au dépôt et à la diffusion de documents scientifiques de niveau recherche, publiés ou non, émanant des établissements d'enseignement et de recherche français ou étrangers, des laboratoires publics ou privés. 


\title{
GEOMETRIC BASED 3D FACIAL GENDER CLASSIFICATION
}

\author{
Lahoucine Ballihi ${ }^{1,2}$, Boulbaba Ben Amor ${ }^{1,3}$, Mohamed Daoudi ${ }^{1,3}$, Anuj Srivastava ${ }^{4}$, Driss Aboutajdine ${ }^{2}$ \\ ${ }^{1}$ LIFL (UMR USTL/CNRS 8022), Universite de Lille1, 59650 Villeneuve d'Ascq France. \\ ${ }^{2}$ LRTT, Unite Associee au CNRST (URAC 29), Universite Mohammed V - Agdal, Rabat, Maroc. \\ ${ }^{3}$ Institut TELECOM ; TELECOM Lille 1, 59650 Villeneuve d'Ascq France. \\ ${ }^{4}$ Departement of Statistics, Florida State University, Tallahassee, FL 32306, USA.
}

\begin{abstract}
This paper addresses the issue of Gender Classification from 3D facial images. While most of previous work in the literature focuses on either 2D facial images, here, we study the use of 3D facial shape for automatic gender classification. After a preprocessing step to extract the facial masks from triangular meshes obtained using laser range scanners, we approximate the facial surfaces by collections of radial and iso-level curves. Once the curves are extracted, we aim at studying their shape using existant shape analysis framework which allows to compute similarities between a candidate face and Male and Female templates. We expect that the shape of certain curves are similar within Male/Female classes and different when moving from one class to another. For classification, we perfom three Machine Learning algorithms (Adaboost, Neural Network, and SVM). Overall, Adaboost was superior in classification performance $\mathbf{( 8 4 . 9 8 \%}$ as classification rate) on a subset of FRGCv2 dataset including the first (neutral and non-neutral) scans of different subjects. Our results indicate also that (i) the most relevant iso-level curves cover the central stripe of the face, and (ii) the most relevant radial curves are located on the upper part of the face.
\end{abstract}

Index Terms - Gender classification, Riemannian geometry, shape analysis, boosting.

\section{INTRODUCTION}

Facial gender is one of the most visual tasks used by humans to help identification and make interaction with other people. In computer vision, gender classification could be a useful preprocessing step for face recognition as other soft biometric traits like skin color, age, eyes colors, and so on used by humans to distinguish their peers. Most existing work use 2D-images to extract distinctive facial features like hair density and inner morphology of the face. While academic and industrial interest in $3 \mathrm{D}$ face recognition has grown in recent years due to its theoretical robustness to lighting condition and pose variations, few approaches try to use 3D images, complementary to $2 \mathrm{D}$ ones, to make gender classification. Many techniques have been proposed for solving the gender classification problem, however most of them are based on 2D face image, only a few studies have investigated on 3D facial shape. Bruce et al. [1] made an interesting experiment in which they tested the human visual system which are remarkably accurate (approaching ceiling) at deciding whether faces are male or female, even when cues from hairstyle, makeup, and facial hair are minimized. The authors have obtained that the subjects were considerably less accurate when asked to judge the sex of three-dimensional (3D) representations of faces obtained by laser-scanning, compared with a condition where photographs were taken with hair concealed and eyes closed. In $3 \mathrm{D}$, they approved that the average male face differs from the average female face by having a more protuberant nose/brow and more prominent chin/jaw. The effects of manipulating the shapes of the noses and chins of the laser-scanned heads were assessed and significant effects of such manipulations on the apparent masculinity or femininity of the heads were revealed. In [2] O'Toole et al. have supposed that the sex of a face is perhaps its most salient feature. They applied a principal components analysis (PCA) separately to the three-dimensional structure and graylevel image data from laser-scanned human heads. The results indicated that the three-dimensional head data supported more accurate sex classification than the graylevel image data, across a range of PCAcompressed (dimensionality-reduced) representations of the heads. Yuan et al. [3] proposed a novel fusion-based gender classification method that is able to compensate for facial expression. They performed experimental investigation to evaluate the significance of different facial regions in the task of gender classification. Jing et al. [4] were investigated gender classification based on 2.5D facial surface normals (facial needle-maps) which can be recovered from 2D intensity images using a non-lambertian Shape-from-shading (SFS) method. The authors described a weighted principal geodesic analysis (WPGA) method to extract features from facial surface normals to increase the gender discriminating power in the leading eigenvectors. They adopted an a posteriori probability based method for gender classification. Xiaoguang et al. [5] exploited the range information of human faces for ethnicity identification using a support vector machine. An integration scheme is also proposed for ethnicity and gender identifications by combining the registered range and intensity images. The $3 \mathrm{D}$ images provides competitive discriminative power on ethnicity and gender identifications to the intensity modality is demonstrated. To the best of our knowledge, no approaches have been proposed to exploit 3D facial curves where shape analysis of 3D face return. But, does the use of all the curves on the face lead to better gender classification performances?, and among the facial curves is there any ones more relevant than others to discriminate gender from facial surface?

In this work, we focus on the geometric shape analysis of the 3D face followed by using machine learning techniques to build classifiers. The basic idea is to approximate a facial surface by a finite set of iso-level curves and radial curves. Using Riemannian geometry we define geodesic paths between facial curves, and distances between them. Feature vectors for classification are produced by comparing test faces to arbitrary male and female templates. Feature vectors are used as input of machine learning techniques. 


\section{OUTLINE OF THE PROPOSED APPROACH}

We propose a fully automatic method to perform gender classification based on 3D face. Our approach combines geometric shape analysis of facial curves with Machine Learning techniques. Figure 1 gives an overview of the proposed approach. After 3D scan acquisition and preprocessing in order to extract the informative area of the face (facial mask) and correct some imperfections such as filling holes and removing noise, we extract both radial and iso-level facial curves from the $3 \mathrm{D}$ surface. Then, according to the nature of the curves (close or open), we employ one of the adapted geometric shape analysis frameworks described in [6] to analyse then compare shapes of corresponding curves on a candidate face and a Male/Female face templates. This allows to form feature vector for each face candidate. The Off-line training step perform a learning step using one of three classical machine learning techniques (Adaboost, Neural Network, and SVM) to build classifiers. In the Online gender classification step, computes vector features for a face candidate and use one of the three classifier to make the decision.

The present paper adopts the following organization: Geometric shape analysis and comparison for both open curves and close curves procedures are explained in section 3 . In section 4 , we detail the Boosting procedure to select the most discriminative facial curves and build strong classifier for gender classification. Experimental evaluations are given in section 5 . The last section gives some concluding remarks and open some future directions.

\section{GEOMETRIC SHAPE ANALYSIS OF FACIAL CURVES}

We start by considering a facial curve $\beta$ in $\mathbb{R}^{3}$. It is natural to parametrize it using $\beta: \mathbb{S}^{1} \rightarrow \mathbb{R}^{3}$. Note that the parameterization is not assumed to be arc-length; we allow a larger class of parameterizations for improved analysis. To analyze the shape of $\beta$, we shall represent it mathematically using a square-root velocity function (SRVF), proposed in $[7,6]$, and denoted by $q(t)$, according to:

$$
q(t) \doteq \frac{\dot{\beta}(t)}{\sqrt{\|\dot{\beta}(t)\|}}
$$

Where $\|$.$\| is the Euclidean norm and q(t)$ is a special function that captures the shape of $\beta$ and is particularly convenient for shape analysis, as we describe next. The conventional metric for comparing the elastic shape of the curves becomes an $\mathbb{L}^{2}$ metric under the representation $[7,6]$. Depends on the topology of the curve (close or open), we recall following shape analysis foundations of facial curves.

\subsection{Radial open curves}

We define the set of open curves in $\mathbb{R}^{3}$ by $\mathcal{C}=\left\{q: I \rightarrow \mathbb{R}^{3} \mid\|q\|=\right.$ 1) $\subset \mathbb{L}^{2}\left(I, \mathbb{R}^{3}\right)$, with the $\mathbb{L}^{2}$ metric on its tangent spaces, $\mathcal{C}$ becomes a Riemannian manifold. In particular, since the elements of $\mathcal{C}$ have a unit $\mathbb{L}^{2}$ norm, $\mathcal{C}$ is a hypersphere in the Hilbert space $\mathbb{L}^{2}\left(I, \mathbb{R}^{3}\right)$. In order to compare the shapes of two radial curves, we can compute the distance between them in $\mathcal{C}$ under the chosen metric. This distance is defined to be the length of the (shortest) geodesic connecting the two points in $\mathcal{C}$. Since $\mathcal{C}$ is a sphere, the formulas for the geodesic and the geodesic length are already well known. The geodesic length between any two points $q_{1}, q_{2} \in \mathcal{C}$ is given by:

$$
d_{c}\left(q_{1}, q_{2}\right)=\cos ^{-1}\left(\left\langle q_{1}, q_{2}\right\rangle\right) \text {, }
$$

and the geodesic path $\alpha:[0,1] \rightarrow \mathcal{C}$, is given by:

$$
\alpha(\tau)=\frac{1}{\sin (\theta)}\left(\sin ((1-\tau) \theta) q_{1}+\sin (\theta \tau) q_{2}\right),
$$

where $\theta=d_{c}\left(q_{1}, q_{2}\right)$

It is easy to see that several elements of $\mathcal{C}$ can represent curves with the same shape. For example, if we rotate a face in $\mathbb{R}^{3}$, and thus its facial curves, we get different SRVFs for the curves but their shapes remain unchanged. Another similar situation arises when a curve is re-parametrized; a re-parameterization changes the SRVF of curve but not its shape. In order to handle this variability, we define orbits of the rotation group $S O(3)$ and the re-parameterization group $\Gamma$ as equivalence classes in $\mathcal{C}$. Here, $\Gamma$ is the set of all orientationpreserving diffeomorphisms of $I$ (to itself) and the elements of $\Gamma$ are viewed as re-parameterization functions. For example, for a curve $\beta: I \rightarrow \mathbb{R}^{3}$ and a function $\gamma \in \Gamma$, the curve $\beta_{\alpha} \circ \gamma$ is a re-parameterization of $\beta_{\alpha}$. The corresponding SRVF changes according to $q(t) \mapsto \sqrt{\dot{\gamma}(t)} q(\gamma(t))$. We define the equivalent class containing $q$ as:

$$
[q]=\{\sqrt{\dot{\gamma}(t)} O q(\gamma(t)) \mid O \in S O(3), \quad \gamma \in \Gamma\},
$$

The set of such equivalence class is called the shape space $\mathcal{S}$ of elastic curves [7]. To obtain geodesics and geodesic distances between elements of $\mathcal{S}$, one needs to solve the optimization problem. The resulting shape space is the set of such equivalence classes:

$$
\mathcal{S} \doteq \mathcal{C} /(S O(3) \times \Gamma)
$$

We denote by $d_{s}\left(\beta_{\alpha_{1}}, \beta_{\alpha_{2}}\right)$ the geodesic distance between the corresponding equivalence classes $\left[q_{1}\right]$ and $\left[q_{2}\right]$ in $\mathcal{S}$. The figure 2 illustrates some examples of geodesic paths between facial surfaces and associated collections of radial curves. The first two rows give an intra-class (same person, different expressions) geodesic path, whereas the remaining two rows show an inter-class (different subjects) geodesic path.

\subsection{Iso-level closed curves}

In the same manner, we define the set of closed curves in $\mathbb{R}^{3}$ by $\left.\tilde{\mathcal{C}}=\left\{q: \mathbb{S}^{1} \rightarrow \mathbb{R}^{3} \mid \int_{\mathbb{S}^{1}} q(t)\|q(t)\| d t=0\right\} \subset \mathbb{L}^{2}\left(\mathbb{S}^{1}, \mathbb{R}^{3}\right)\right\}$, where $\mathbb{L}^{2}\left(\mathbb{S}^{1}, \mathbb{R}^{3}\right)$ denotes the set of all integrable functions from $\mathbb{S}^{1}$ to $\mathbb{R}^{3}$. The quantity $\int_{\mathbb{S} 1} q(t)\|q(t)\| d t$ is the total displacement in $\mathbb{R}^{3}$ while moving from the origin of the curve until the end. When it is zero, the curve is closed. Thus, the set $\tilde{\mathcal{C}}$ represents the set of all closed curves in $\mathbb{R}^{3}$. It is called a pre-shape space since curves with same shapes but different orientations and re-parameterizations can be represented by different elements of $\tilde{\mathcal{C}}$. To define a shape, its representation should be independent of its rotations and reparameterization. This is obtained mathematically by a removing the rotation group $S O(3)$ and the re-parameterization group $\Gamma$ from $\tilde{\mathcal{C}}$. As described in $[7,6]$, we define the orbits of the rotation group $S O(3)$ and the re-parameterization group $\Gamma$ as equivalence classes in $\tilde{\mathcal{C}}$. The resulting shape space is :

$$
\tilde{\mathcal{S}} \doteq \tilde{\mathcal{C}} /(S O(3) \times \Gamma)
$$

To define geodesics on pre-shape and shape spaces we need a Riemannian metric. For this purpose we inherit the standard $\mathbb{L}^{2}$ metric the large space $\mathbb{L}^{2}\left(\mathbb{S}^{1}, \mathbb{R}^{3}\right)$. For any $u, v \in \mathbb{L}^{2}\left(\mathbb{S}^{1}, \mathbb{R}^{3}\right)$, the standard $\mathbb{L}^{2}$ inner-product is given by:

$$
\langle\langle u, v\rangle\rangle=\int_{\mathbb{S} 1}\langle u(t), v(t)\rangle d t .
$$




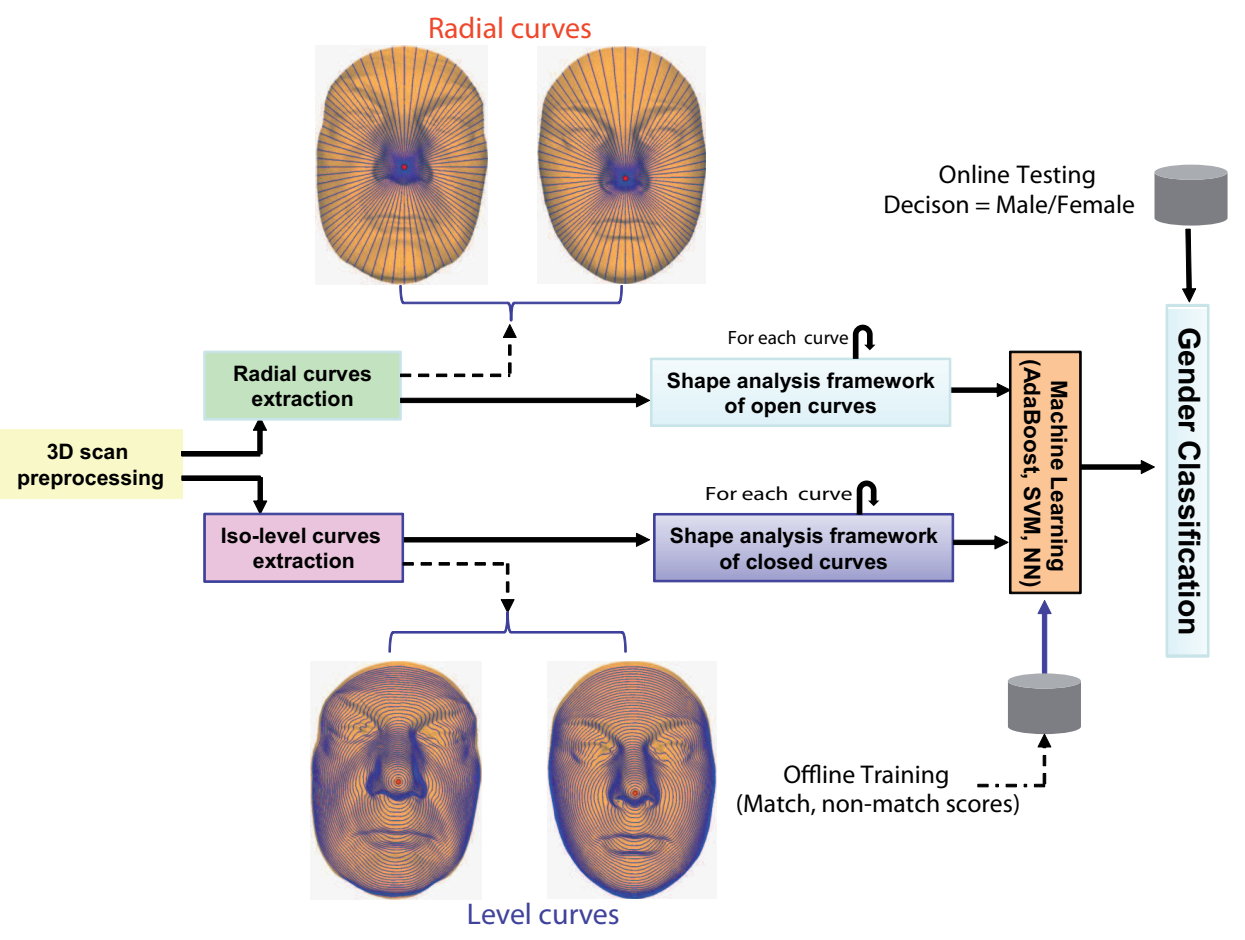

Fig. 1: Outline of the proposed 3D face gender classification approach.

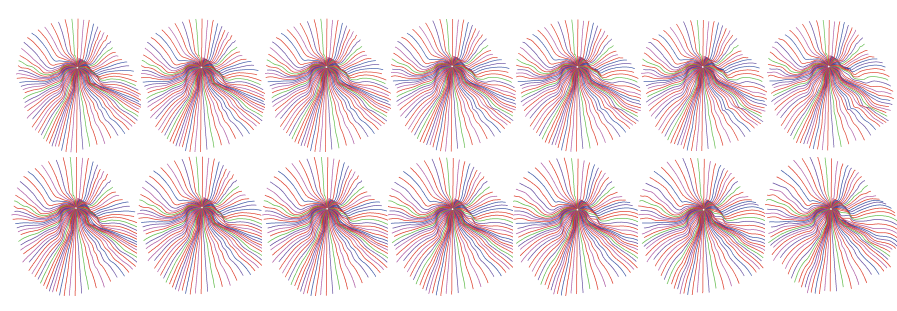

Fig. 2: Examples of intra- and inter-class geodesics between facial surfaces and their associated radial curves.

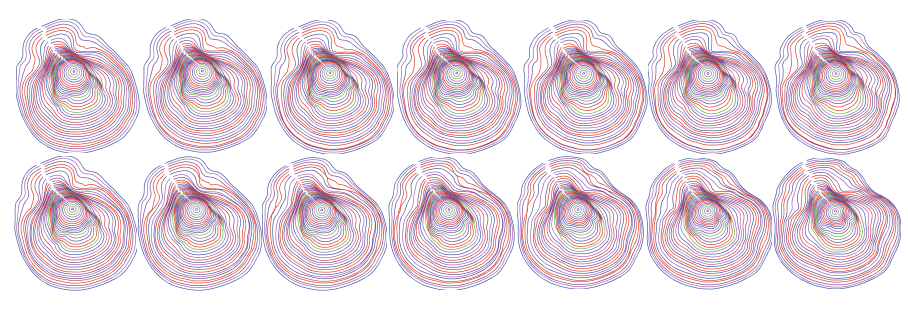

Fig. 3: Examples of intra- and inter-class geodesics between facial surfaces and their associated iso-level curves.

The computation of geodesics and geodesic distances utilize the intrinsic geometries of these spaces. While the detailed description of the geometries of $\tilde{\mathcal{C}}$ and $\tilde{\mathcal{S}}$ are given in [7, 6]. An important tool in our framework is the construction of a geodesic path between two elements of $\tilde{\mathcal{S}}$, under the Riemannian metric given by equation 5 .
Given two curves $\beta_{\lambda_{1}}$ and $\beta_{\lambda_{2}}$, represented by their SRVF respectively $q_{1}$ and $q_{2}$, we need to find a geodesic path between the orbits $\left[q_{1}\right]$ and $\left[q_{2}\right]$ in the space $\tilde{\mathcal{S}}$. We use in this context, a numerical method called the path-straightening method [8] which connects the two points $\left[q_{1}\right]$ and $\left[q_{2}\right]$ an arbitrary path $\alpha$ and then updates this path repeatedly in the negative direction of the gradient of energy given by:

$$
E[\alpha]=\frac{1}{2} \int_{s}\langle\langle\dot{\alpha}(s), \dot{\alpha}(s)\rangle\rangle d s
$$

It has been proven in [8] that the critical points of $E$ are geodesic paths in $\tilde{\mathcal{S}}$. We denote by $d_{\tilde{s}}\left(\beta_{\lambda_{1}}, \beta_{\lambda_{2}}\right)$ the geodesic distance between the corresponding equivalence classes $\left[q_{1}\right]$ and $\left[q_{2}\right]$ in $\tilde{\mathcal{S}}$. The figure 3 illustrates some examples of geodesic paths between facial surfaces and associated collections of iso-level curves. The first two rows give an intra-class (same person, different expressions) geodesic path, whereas the remaining two rows show an inter-class (different subjects) geodesic path.

\section{BOOSTING FOR GENDER CLASSIFICATION}

Radial and iso-level curves capture locally the shape of different facial regions. However, to recognize the gender of facial surfaces, we only interested to the shapes of some facial regions. In gender classification application, the comparison of these curves shapes, runs into trouble according to the degree of facial regions shapes (male/female) changes. For that purposes, we introduce a feature selection step to identify (or localize) the most discriminative curves. We propose to use the well-known machine learning algorithm, AdaBoost, introduced by Freund and Schapire in [9]. Boosting is based on iterative selection of weak classifiers by using a distribution of training samples. At each iteration, the best (relevant) weak classifier 


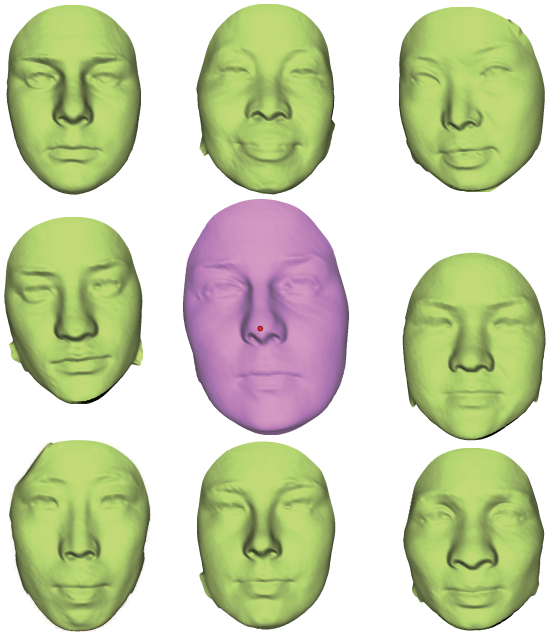

Fig. 4: Examples of females facial surfaces and the template face is shown in the center of figure

is provided and weighted by the quality of its classification. In practice, the individual iso-level curves and radial curves are used as the weak classifiers. After $M$ iterations, the most relevant $T(T<M)$ facial curves are returned by the algorithm.

To train and test the boosting algorithm for this application, we use all scans of all subjects present in FRGCv2 dataset(466). Firstly we selected the first male scan as male facial template that we called $F_{\text {temp }}^{\text {male }}$ while the first female scan as female facial template, $F_{\text {temp }}^{\text {female }}$.

For each face candidate, we compute the distance over all curves to the defined templates. This allows to form feature vectors for each test face. Let (i) match scores distances when face candidate and the template belong to the same class, and (ii) non-match scores are distances between candidate face and template when belong to different classes. In these areas of the matrices, we extract two kind of scores (i) the match scores (intra-class comparisons) and (ii) the non-match scores (inter-class comparison).

Table 1: Match scores and non-match scores for Machine Learning inputs.

\begin{tabular}{||c||c||c||c|}
\hline For each curve & Face male1 & Face female1 & $\ldots$ \\
\hline$F_{\text {temp }}^{\text {male }}$ & $\left\{x_{n}^{\alpha, \lambda}, 1\right\}$ & $\left\{x_{n}^{\alpha, \lambda}, 0\right\}$ & $\ldots$ \\
\hline$F_{\text {temp }}^{\text {temale }}$ & $\left\{x_{n}^{\alpha, \lambda}, 0\right\}$ & $\left\{x_{n}^{\alpha, \lambda}, 1\right\}$ & $\ldots$ \\
\hline
\end{tabular}

Both scores lists represent the input of the machine learning. More formally, we consider a set of pairs $\left(x_{n}^{\alpha, \lambda}, y_{n}\right)_{1 \leq n \leq N}$ where $x_{n}^{\alpha, \lambda}$ is a similarity score between two curves at the same level $\alpha, \lambda$ and $y_{n}$ can take two values: 0 in the case of match score and 1 in the case of non-match score. For each curve $\beta_{j}$, the weak learner determines the optimal threshold classification function, such that the minimum number of scores are misclassified. A weak classifier $h_{j}\left(x_{n}^{k}\right)$ thus consists of a geometric feature $\beta_{j}$ and a threshold $\theta$, such that

$$
h_{j}\left(x_{n}^{k}\right)= \begin{cases}1 & \text { if } x_{n}^{k}<\theta \text { (intra-class) } \\ 0 & \text { otherwise. (inter-class) }\end{cases}
$$

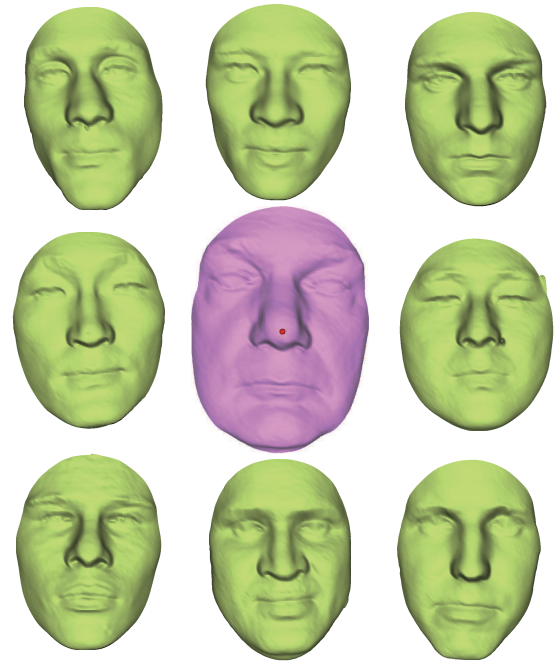

Fig. 5: Examples of males facial surfaces and the template face is shown in the center of figure

The figure 5 consists of some facial surfaces of different males persons taken from FRGCv2, and the template face is shown in the center of the figure, while the Figure 4 shows some different females persons taken from FRGCv2, and the template face is shown in the center of the figure.

\section{EXPERIMENTAL RESULTS}

The difficulty encountered to compare our approach with those published in the state of the art, unfortunately so far there is no standard protocol to compare gender classification results, contrary to the face recognition. The most state-of-the-art approaches ([3],[4]) use the selected databases to publish the results of their approaches. In contrast, we will give the first result of gender classification on the the will known database FRGCv2 and also use all subjects 466 .

Experiments have been carried out on a subset taken from the FRGCv2 dataset including the first (neutral and non-neutral) scans of different subjects, 466 individual in total. Face scans are given as matrices of size $480 \times 640$ of 3D points, individuals have been acquired with near-frontal view. The Adaboost algorithm has been trained and tested using 10-fold cross validation. According to this, the dataset is split into a training and a test subset of size $1 / 10$ and $9 / 10$ of the original dataset, respectively. Training is repeated 10 times, with each of the 10 subsets used exactly once as the test data. Finally, the results from the ten steps are averaged to produce a single estimation of the performance of the classifier. In this way, all observations are used for both training and test, and each observation is used for test exactly once. The table 2 gives gender classification results for the three classification methods Adaboost, Neural Network, and SVM.

As shown in this table, the Boosting outperforms Neural Network and SVM algorithms. It achieves near $85 \%$ as correct classification which is an encouraging result. To understand and explain the results mentioned above, we looked at the early iterations of the Boosting algorithm and the selected radial and iso-level curves in each iteration. Figures 6 and 7 gives the locations of most relevant 
Table 2: Gender classification results using different Adaboost, Neural Network, and SVM.

\begin{tabular}{|c|c|c|c|}
\hline \multirow{2}{*}{} & \multicolumn{3}{|c|}{ Machine Learning Methods } \\
\cline { 2 - 4 } & AdaBoost $^{(*)}$ & $\mathrm{SVM}^{(*)}$ & $\mathrm{NN}^{(*)}$ \\
\hline \hline Gender Classification Rate & $84.98 \%$ & $83.69 \%$ & $84.33 \%$ \\
\hline
\end{tabular}

(*) Results for 10-fold cross-validation.

curves on both male and female surfaces.
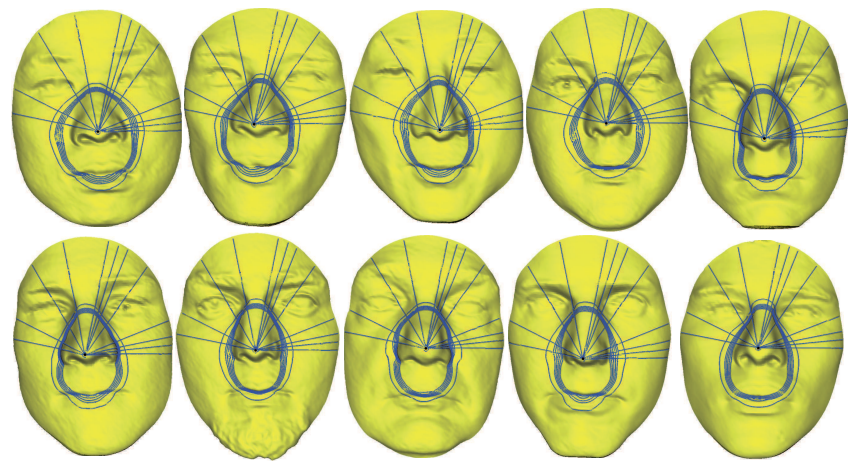

Fig. 6: Relevant radial and iso-level curves selected by Boosting given on different male faces.

We can notice that (i) Selected iso-level curves are located on the central stripe of the face. This is due to the different male/female faces morphology, especially the volume of the nose which is, in general, more significant for males than females as explained in [1]. (ii) Selected iso-level curves are located on the upper part of the face and pass throw the cheek/nose/brow regions. These regions present different shapes between the two classes.
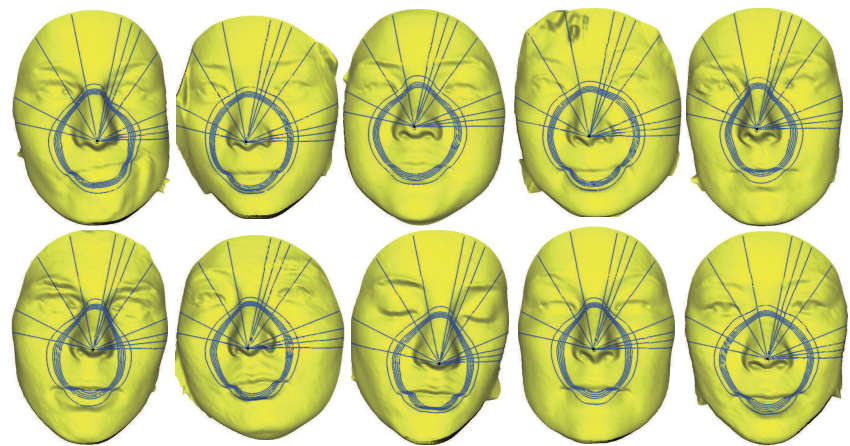

Fig. 7: Relevant radial and iso-level curves selected by Boosting given on different female faces.

\section{CONCLUSION}

In this paper we demonstrate the effectiveness of the use of 3D information for gender classification. First, we consider both radial and iso-level curves which are significantly used in the 3D face recognition literature. Second, by using existing tools for facial shape analysis and comparison, we compare candidate faces to male and female facial templates over all curves to produce feature vectors. Then these vectors will be considered as inputs of Machine Learning techniques (Boosting, Neural Network, and SVM) to build classifiers. We showed in our experimental results that the classifiers achieved comparable results and by examinating the (relevant) selected curves are located in facial regions where face morphology is different. We demonstrated that the use of 3D shape of face could be a powerful analysis tool to study the facial variation with sex.

As future directions, the presented shape analysis framework allows to compute statistics as the intrinsic mean (average) shape which could be interesting to build more representative templates of male/female classes. We try also to use same approach to study more classification problem as ethnicity classification (Caucasian/Asian) or facial expression recognition.

\section{REFERENCES}

[1] V Bruce, A M Burton, E Hanna, P Healey, O Mason, A Coombes, R Fright, and A Linney, "Sex discrimination: how do we tell the difference between male and female faces?," Perception, vol. 22, no. 2, pp. 131-52, 1993.

[2] A J O'Toole, T Vetter, N F Troje, and H H Bülthoff, "Sex classification is better with three-dimensional head structure than with image intensity information.," Perception, vol. 26, no. 1, pp. 75-84, 1997.

[3] Li Lu and Pengfei Shi, "A novel fusion-based method for expression-invariant gender classification," in ICASSP, 2009, pp. $1065-1068$.

[4] Jing Wu, William A. P. Smith, and Edwin R. Hancock, "Facial gender classification using shape from shading and weighted principal geodesic analysis," in ICIAR, 2008, pp. 925-934.

[5] Xiaoguang Lu, Hong Chen, and Anil K. Jain, "Multimodal facial gender and ethnicity identification," in ICB, 2006, pp. 554561.

[6] A. Srivastava, E. Klassen, S. H. Joshi, and I. H. Jermyn, "Shape analysis of elastic curves in euclidean spaces," IEEE Transactions on Pattern Analysis and Machine Intelligence, vol. 33, no. 7, pp. 1415-1428, 2011.

[7] S. H. Joshi, E. Klassen, A. Srivastava, and I. H. Jermyn, "A novel representation for efficient computation of geodesics between $n$-dimensional curves," in IEEE CVPR, 2007, pp. 1-7.

[8] E. Klassen and A. Srivastava, "Geodesics between 3D closed curves using path-straightening," in Proceedings of ECCV, Lecture Notes in Computer Science, 2006, pp. I: 95-106.

[9] Y. Freund and R. E. Schapire, "A decision-theoretic generalization of on-line learning and an application to boosting," in EuroCOLT '95: Proceedings of the Second European Conference on Computational Learning Theory, London, UK, 1995, pp. 23-37, Springer-Verlag. 